\title{
The potential contribution of preplanned refixations to the preferred viewing location
}

\author{
SCOTT A. McDONALD and RICHARD C. SHILLCOCK \\ University of Edinburgh, Edinburgh, Scotland
}

\begin{abstract}
On the basis of recent evidence for the preplanning of refixation saccades in reading-like tasks (e.g., Beauvillain, Vergilino, \& Dukic, 2000), we proposed that for a proportion of words refixated during natural reading, the refixation sequence is planned before the word is fixated, and that the saccade target for these cases is near the beginning of the word. The preferred viewing location (PVL) phenomenon can then be explained as a mixture of two populations of planned saccades: those that initiate a multiplefixation sequence and single fixations. This account supplants saccadic undershooting as the primary determinant of the PVL. We used a corpus of eye movement data to show that the probability of refixation is strongly correlated with the estimated perceptibility of the target word from the current fixation location, and that the observed (re-)fixation behavior is justified when assessed in terms of a lexical uncertainty measure. Refixations may be preplanned in order to increase the chances of successful word identification.
\end{abstract}

The preferred viewing location (PVL) is a term originated by Rayner (1979) to describe the position in a word where the eyes first land during reading. A number of eyetracking studies have shown that readers tend to make their initial fixation between the beginning and middle of the word, whether they are reading left-to-right orthographies such as English (e.g., Dunn-Rankin, 1978; McConkie, Kerr, Reddix, \& Zola, 1988; O’Regan, 1981; Rayner, 1979; Vitu, 1991b; Vitu, McConkie, Kerr, \& O’Regan, 2001) or right-to-left languages such as Hebrew (Deutsch \& Rayner, 1999).

The PVL is visually apparent when the relative frequency distributions of initial fixation locations are plotted for length-segregated words. Figure 1 displays the landing position distributions for words of 4-8 letters in length according to the data from the EMBRA eye movement corpus (see the Method section for a description of this corpus). The probability of the eyes initially fixating a given letter position is estimated by the relative frequency in the corpus. These data exhibit the typical properties of the landing position distributions that have been reported by other researchers (e.g., McConkie et al., 1988; Rayner, 1979; Vitu et al., 2001). First, the curves for shorter words are flatter than those for longer words (the mode of the curve is more easily distinguished for longer words); second, the majority of the probability mass is distrib-

This work was supported by Project Grant GR064240AIA from the Wellcome Trust. R.C.S. was also supported by a Senior Research Fellowship from the Economic and Social Research Council. We are grateful to Padraic Monaghan and Sarah White for useful feedback on an earlier version of this article. Correspondence should be addressed to S. A. McDonald, Department of Psychology, University of Edinburgh, 7 George Square, Edinburgh EH8 9JZ, Scotland (e-mail: scott.mcdonald (a)ed.ac.uk). uted over the first half of the word. The PVL phenomenon is apparent for longer (5- to 8-letter) words: the "preferred" initial landing position is between the beginning and middle of the word. The mean landing positions for 4- through 10-letter words are provided in Table 1; for all word lengths, the mean is between word-beginning and word-center. It is also clear from Table 1 that the (meansummarized) PVL varies according to word length, moving rightward for longer words.

As first pointed out by McConkie et al. (1988), plots such as Figure 1 are actually best described as composite landing position distributions because the curves can be decomposed as a function of launch distance (the distance, in character spaces, from the previous fixation to the target word). Composite landing position distributions obscure a strong effect of launch distance that McConkie et al. (1988) attribute to a systematic range error: undershooting far targets and overshooting near ones (Kapoula, 1985; Kapoula \& Robinson, 1986). Assuming that the center of a word is the saccade target, saccades launched from more than approximately 7 character spaces in front of word-center tend to undershoot the target location, and saccades launched from fewer than 7 character spaces tend to overshoot the target. This range error is on the order of $0.35-0.5$ characters per character shift of launch distance (McConkie et al., 1988; Radach \& McConkie, 1998). McConkie et al. (1988) demonstrate that for a center-based launch distance of about 7 characters, the mean of the resulting landing position distribution is located near wordcenter.

Saccadic undershooting forms the basis of the generally accepted explanation for the PVL. This explanation rests on two premises, supported by the oculomotor literature (see, e.g., Becker, 1989); first, that saccades are aimed at or near the middle of the target word; and sec- 


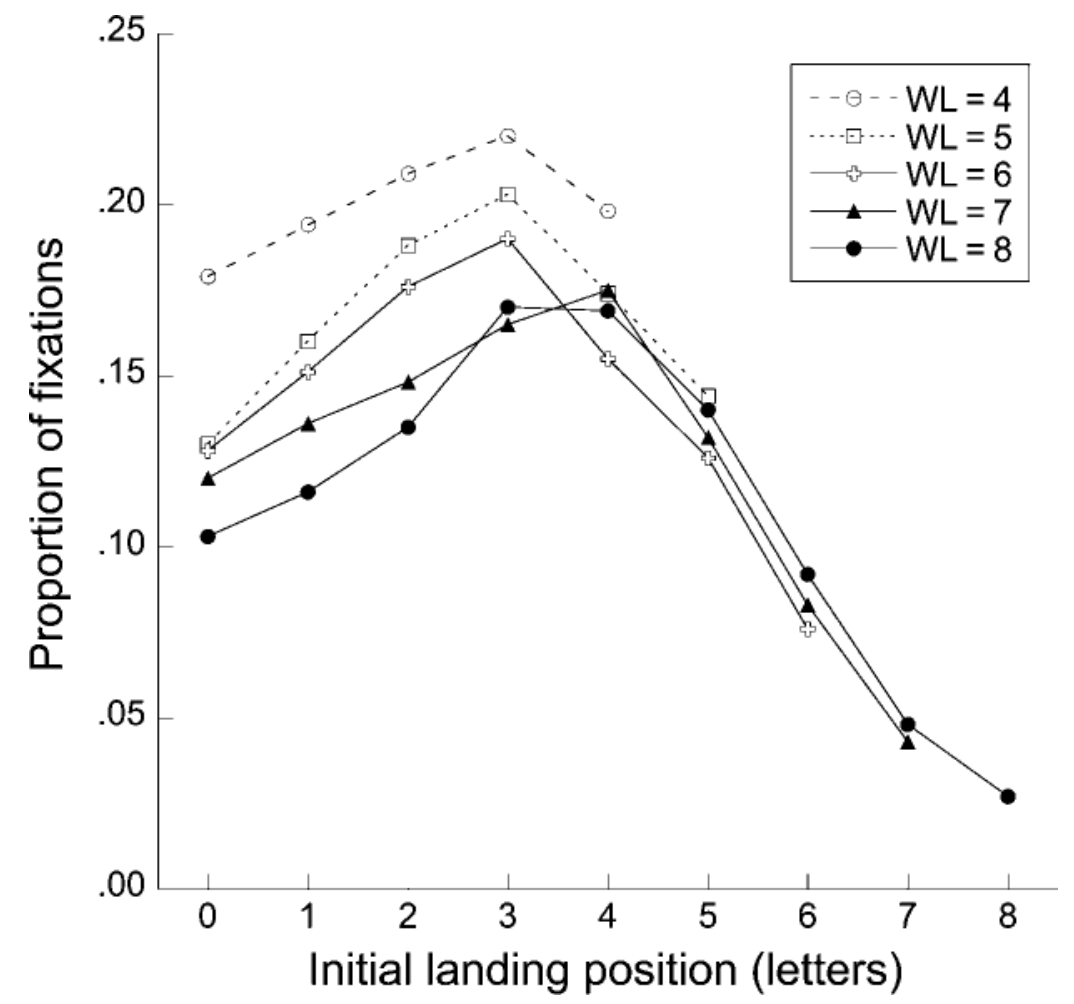

Figure 1. Composite landing position distributions for 4- to 8-letter words. Data are aggregated over 23 subjects each reading approximately 2,300 words of newspaper text.

ond, that saccades tend to fall short of this target, landing between word-beginning and word-center. Composite landing position curves, such as those plotted in Figure 1, are consistent with this view, showing us that, on average, saccades tend to land short of word-center. Undershooting the target location presumably must be more prevalent than overshooting. Our eye movement corpus data are also consistent with an undershooting explanation for the PVL. The average launch distance for the 4- to 10letter words in our corpus is 6.8 characters before the space before the target word (see Table 1), corresponding

Table 1

Mean Initial Landing Position (in Letters) and Mean Launch Distance (in Character Spaces Before the Space in Front of the Target Word), With Standard Deviations, and Refixation Proportion, for 4- to 10-Letter Words

\begin{tabular}{|c|c|c|c|c|c|}
\hline \multirow{2}{*}{$\begin{array}{c}\text { Word } \\
\text { Length }\end{array}$} & \multicolumn{2}{|c|}{ Landing Position } & \multicolumn{2}{|c|}{ Launch Distance } & \multirow{2}{*}{$\begin{array}{c}\text { Proportion } \\
\text { Refixated }\end{array}$} \\
\hline & $M$ & $S D$ & $M$ & $S D$ & \\
\hline 4 & 2.06 & 1.38 & 7.11 & 3.99 & .052 \\
\hline 5 & 2.56 & 1.60 & 6.68 & 3.82 & .073 \\
\hline 6 & 2.77 & 1.79 & 6.80 & 3.99 & .102 \\
\hline 7 & 3.08 & 1.98 & 6.51 & 4.24 & .128 \\
\hline 8 & 3.37 & 2.09 & 6.55 & 3.94 & .131 \\
\hline 9 & 3.62 & 2.28 & 6.66 & 4.15 & .156 \\
\hline 10 & 3.74 & 2.27 & 6.65 & 4.31 & .230 \\
\hline
\end{tabular}

to an average of 10.3 character spaces before word-center. Hence, the PVL could simply be due to the average launch distance being longer than the reported 7 characters (McConkie et al., 1988), which centers the landing position distribution over the target word.

Although other explanations for the PVL phenomenon have been proposed, we will not consider them in further detail here. For instance, several researchers have suggested that the center of gravity of the visual configuration influences the initial fixation location (e.g., Findlay, 1982; Vitu, 1991a). Ducrot and Pynte (2002) attribute the PVL effect to attentional processes invoked for the processing of discrete stimuli. Lexical informativeness has also been proposed as a factor affecting landing position (e.g., Hyönä, 1995; G. Underwood, Clews, \& Everatt, 1990; but cf. Rayner \& Morris, 1992): in many languages, the beginning of a word is more informative about its identity than its ending (cf. Shillcock, Ellison, \& Monaghan, 2000); thus readers may tend to fixate near word-beginning in order to increase their chances of recognizing the word.

In this article, we put forward the idea that a major part of the preferred viewing location effect is neither an oculomotor nor cognitive phenomenon, but instead can be attributed to a combination of a simple statistical property of landing position distribution data and functional pressure for the eye guidance system to plan re- 
fixation saccades in order to maximize the success of word identification. We discuss empirical evidenceboth from published studies and from analysis of an eye movement corpus collected in our laboratory - that provides an alternative to saccadic undershooting as the best explanation of the PVL effect.

\section{AN ALTERNATIVE VIEW OF REFIXATIONS}

In this section, we consider the proposal that initial landing position distributions represent a mixture of two distinct populations of oculomotor events, and that the PVL is the result of conflating the data from two saccade planning strategies: single fixations and multiple-fixation sequences. This proposal is based on two premises. The first premise is that some proportion of refixations are preplanned; namely, a refixation saccade is programmed before execution of the primary saccade to the target word. The second premise is that the location targeted within the word is not always its center; namely, saccades are aimed at a position near the word-beginning when a further fixation is planned on the target word.

\section{Refixations Can Be Preplanned}

We begin by considering the evidence for and the consequences of the first premise. First, in a series of doublestep experiments using 7- to 11-letter French words and pseudowords, Beauvillain and colleagues (Beauvillain, Vergilino, \& Dukic, 2000; Vergilino \& Beauvillain, 2000; Vergilino-Perez \& Beauvillain, 2004) demonstrated that refixation saccade amplitude was best described as a constant, the value of which was a nearly perfect function of the target word's length. For instance, the amplitude of the refixation saccade did not change if the target string was displaced one or two characters during the initial saccade to the target, suggesting that the refixation saccade was preplanned. The size of the refixation saccade was not affected by the actual landing position in the target word. On the basis of these findings, we suggest that during natural text reading some proportion of refixations are preplanned, where the decision to fixate only once or to refixate is primarily determined by parafoveally obtained visual information about the target word, such as its length. Of course, a number of other factors may trigger a refixation; numerous studies of isolated word reading (e.g., O'Regan, 1990) have shown that refixations are made in situations when preplanning is impossible. ${ }^{1}$ But in the case of natural text reading, the observation that the refixation probability is higher the farther the initial fixation location from word-center (McConkie, Kerr, Reddix, Zola, \& Jacobs, 1989; Vitu, O’Regan, \& Mittau, 1990 ) is also explainable by the planning of a multiplesaccade sequence and may be due to "the preselection of either one or two successive positions within the next word as a function of its length" (Beauvillain et al., 2000, p. 329).

For simplicity, we have referred to the center of a word as the functional target. However, a more behaviorally realistic target location in a word is the optimal viewing po- sition (OVP). The OVP refers to the optimal point of fixation in a word for its recognition when presented in isolation - the location where refixation probability and gaze duration are at a minimum (O'Regan, Lévy-Schoen, Pynte, \& Brugaillère, 1984). On average, the OVP is located slightly left of word-center and to the right of the PVL.

One important consequence of planning a multiplefixation sequence is that the initial saccade may not be directed toward the word-center (or the OVP). For example, if two fixations are planned for a 9-letter target word, the best way to ensure that all of the letters fall upon the fovea (the region of highest visual acuity in the retina: the central $2^{\circ}$ of the visual field) is to make the first fixation near the beginning and the second near the end (see Clark \& O’Regan, 1999, for related discussion).

\section{Initial Fixation Position in Refixated Cases is Almost Constant}

Our second premise, that the within-target location aimed for is not always the word-center, receives some support from Beauvillain et al. (2000, Experiment 2). In this study, participants were required to make a saccade from a fixation cross to a sequence of two 7-letter words. Examination of their data from the control condition where the word sequence was not displaced (see their Figure 6) clearly shows that the modal landing position in the first word was leftward for the refixated cases (between the 2nd and 3rd letters), as compared with the single-fixation cases (between the 3rd and 4th letters). Thus, refixated cases could be distinguished from single-fixation cases by a horizontal shift in the landing position distributions.

In the next section, we present the results of several analyses of a large corpus of eye movement data in order to establish whether preplanned refixations could explain the PVL. We investigated the factors that influence the occurrence of a refixation on the target word and assessed the viability of our hypothesis that the saccade target location is not always the word-center. We then explored a potential motivation for the preplanning of refixations with a simulation procedure.

\section{ANALYSIS OF AN EYE MOVEMENT CORPUS}

\section{Method}

Our analyses employed the EMBRA (Eye Movement Behaviour during the Reading of newspaper Articles) corpus of eye movement data collected in our laboratory. This corpus consists of the eye movement data collected from 23 young adult participants while they each read approximately 2,300 words of contemporary newspaper text presented on a computer monitor. The text consisted of excerpts from 10 articles comprising a broad range of topics. Articles were divided into screens of no more than 10 lines each and were displayed as light cyan characters on a black background. One degree of visual angle was subtended by 3.8 characters. A Fourward Technologies Generation VI DPI eyetracking instrument interfaced to a 486-CPU microcomputer was used to monitor eye movements and collect data. Viewing was binocular, and the position of the right eye was sampled every millisecond. Further details about the apparatus and procedure can be found in McDonald and Shillcock (2003). 
Table 2

Mean Initial Landing Position (in Letters) and Mean Launch Distance (in Character Spaces), With Standard Deviations, for 4- to 10-Letter Words, Comparing Single-Fixation Cases With Refixated Cases

\begin{tabular}{|c|c|c|c|c|c|c|c|c|}
\hline \multirow{3}{*}{$\begin{array}{l}\text { Word } \\
\text { Length }\end{array}$} & \multicolumn{4}{|c|}{ Single-Fixation Cases } & \multicolumn{4}{|c|}{ Refixated Cases } \\
\hline & \multicolumn{2}{|c|}{$\begin{array}{l}\text { Landing } \\
\text { Position }\end{array}$} & \multicolumn{2}{|c|}{$\begin{array}{l}\text { Launch } \\
\text { Distance }\end{array}$} & \multicolumn{2}{|c|}{$\begin{array}{l}\text { Landing } \\
\text { Position }\end{array}$} & \multicolumn{2}{|c|}{$\begin{array}{l}\text { Launch } \\
\text { Distance }\end{array}$} \\
\hline & $M$ & $\frac{S D}{S D}$ & $M$ & $S D$ & $M$ & $\overline{S D}$ & $M$ & $S D$ \\
\hline 4 & 2.07 & 1.37 & 7.07 & 3.99 & 1.98 & 1.59 & 7.92 & 3.96 \\
\hline 5 & 2.61 & $1.5^{\circ}$ & 6.56 & 3.77 & 1.90 & 1.86 & 8.23 & 4.19 \\
\hline 6 & 2.89 & 1.75 & 6.62 & 3.95 & 1.73 & 1.85 & 8.47 & 4.03 \\
\hline 7 & 3.24 & 1.91 & 6.30 & 4.2 & 1.98 & 2.08 & 7.93 & 3.82 \\
\hline 8 & 3.57 & 2.01 & 6.33 & 3.89 & 2.01 & 2.18 & 8.12 & 3.94 \\
\hline 9 & 3.90 & 2.17 & 6.44 & 4.18 & 2.08 & 2.23 & 7.90 & 3.76 \\
\hline 10 & 4.08 & 2.13 & 6.27 & 4.29 & 2.57 & 2.33 & 7.97 & 4.13 \\
\hline
\end{tabular}

For purposes of the analyses reported below, data associated with words occurring at the beginning of a line, words preceded by a punctuation mark, or the first fixation made on a line were excluded from consideration. Fixation data for words on which a track loss, blink, or other signal irregularity occurred were also excluded. After these criteria were met, 16,419 cases were available for analysis $(3,911,3,073,2,952,2,460,1,814,1,402$, and 807 cases, for 4to 10 -letter words, respectively).

\section{Results and Discussion}

The aim of the first analysis was to compare landing position distributions for refixated and single-fixation cases. On the basis of the results of Beauvillain et al.
(2000, Experiment 2), if we also assume that some proportion of the refixations made during natural reading were preplanned, we should also find that landing position distributions in our eye movement corpus differ between refixated and single-fixation cases. The first step involved in testing this prediction was to partition the dataset on the basis of whether or not a refixation was made on the target word. Table 2 provides the mean initial landing positions and launch distances for the separate refixated and single-fixation cases. From Table 2, it is clear that the mean landing position on a refixated word varies very little: Initial fixation position is around the second letter for 4- to 9-letter words, and halfway between the second and third letter of 10-letter words. In contrast, the mean landing position for single-fixation cases moves rightward as a linear function of word length. Importantly, the mean landing position for each of the word lengths considered is slightly left of word-center; thus the PVL effect is attenuated when single-fixation cases are considered separately. This difference is also apparent when the composite landing position distributions for the single-fixation cases only (Figure 2) are compared with the distributions for the aggregate data (Figure 1).

Also of note from Table 2 is that the mean launch site is consistently farther from the target word (approximately 1.7 character spaces) in the refixated cases compared with the single-fixation cases. This launch distance difference would also predict that initial landing positions for the refixated cases tend to be located to the

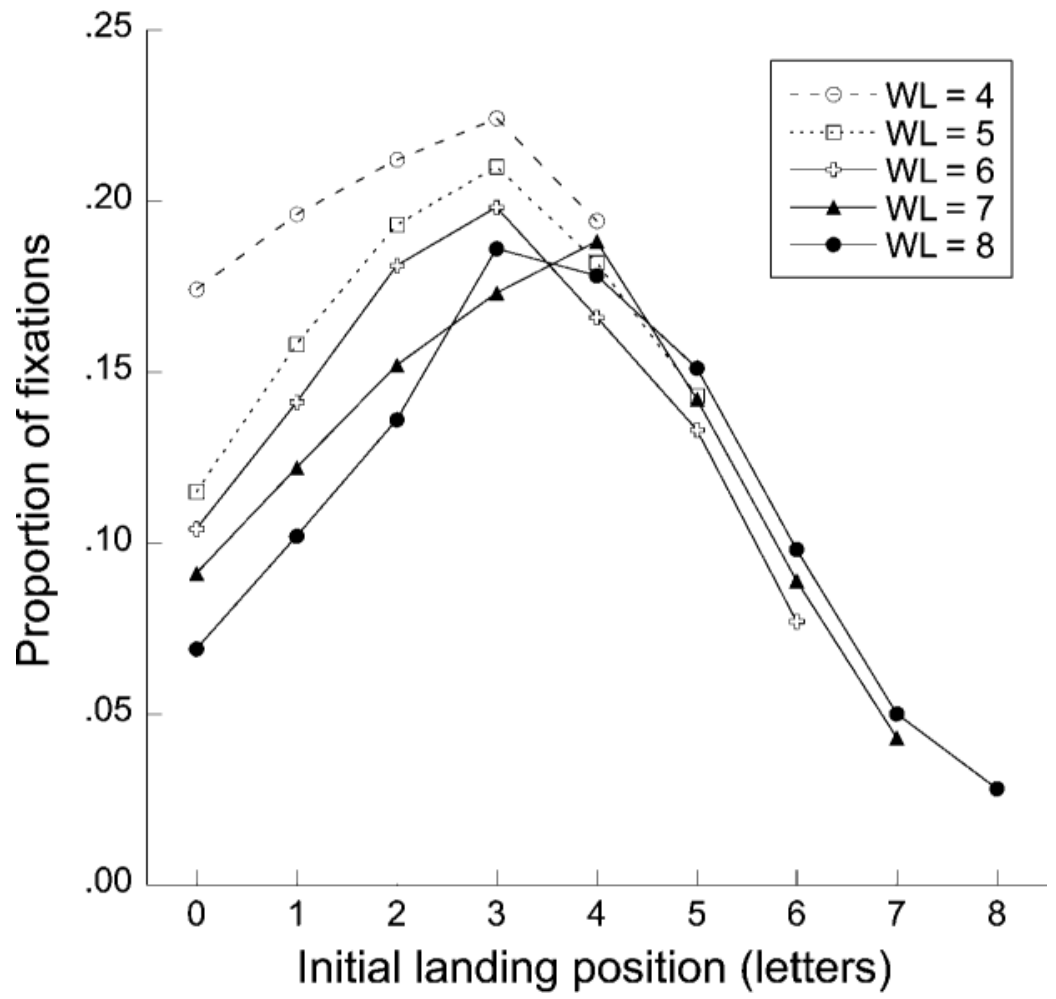

Figure 2. Composite landing position distributions for 4- to 8-letter words receiving a single fixation only. 
left of the landing positions for the single-fixation cases. However, because the mean landing position moves leftward $0.35-0.5$ characters for every 1-character increase in launch distance (Radach \& McConkie, 1998), this launch distance difference could not explain the large landing position differences observed for the longer (7to 10 -letter) words.

In order to further explore the landing position differences between refixated and single-fixation cases, we compared the landing positions of saccades directed toward the target word while holding the launch distance constant. Figure 3 plots this relationship for 6- and 7-letter words (the word lengths for which the most data were available) for launch distances of 1-12 character spaces. First, for the single-fixation cases, the mean landing position moves rightward as a linear function of launch distance, which replicates previous findings (McConkie et al., 1988; Rayner, Sereno, \& Raney, 1996). Second, the refixated cases, although noisier due to a much smaller amount of data, also show a negative relationship between mean landing position and launch distance, indicating that the same linear relationship between these two variables observed for once-fixated words is also present for saccades followed by refixations. The difference in mean initial fixation position between the refixated and singlefixation cases is roughly constant across the launch distance range (the slopes of the regression lines fitted to the two sets of data are very similar). It is clear that the leftward mean landing positions for the refixated cases relative to the single-fixation cases cannot easily be explained as a confound of launch distance. Furthermore, the fact that mean landing position for the refixated cases varies with launch distance is evidence against "direct-control" models of refixation behavior; if the triggering of a refixation saccade de- pended primarily upon initial landing position, there should be no displacement of the mean landing position distribution for refixated cases according to the distance between the current word and the previous fixation.

To summarize our findings thus far, landing position distributions differ between single-fixation and refixation cases, with the mean landing position for the singlefixation cases close to word-center for all word lengths examined. In contrast, there was only a tiny influence of word length on the initial fixation position for the refixated cases: The mean landing position is around the second letter position. We have proposed that for at least some proportion of the refixated cases, saccades were not aimed at the middle of the target word. Instead, our landing position data for refixated words are consistent with a target location near the beginning of the word. In this view, the difference in mean initial fixation position between single-fixation and refixation cases is a consequence and not a cause of refixation planning. The mixing of distributions with distinct saccade targets may constitute a major contribution to the PVL effect observed in composite landing position distributions. Thus, irrespective of any tendency for saccades to undershoot their target, if at least some saccades are aimed at a location near the beginning of the target word, this would account for a substantial portion of the PVL effect.

Our case against saccadic undershooting as the primary determinant of the PVL receives further support from studies examining saccades made against the normal direction of reading. These studies suggest that undershooting is not an inevitable component of saccade behavior during reading. The landing position distributions of interword regressive saccades are centered over the middle of the word (Radach \& McConkie, 1998), irre-
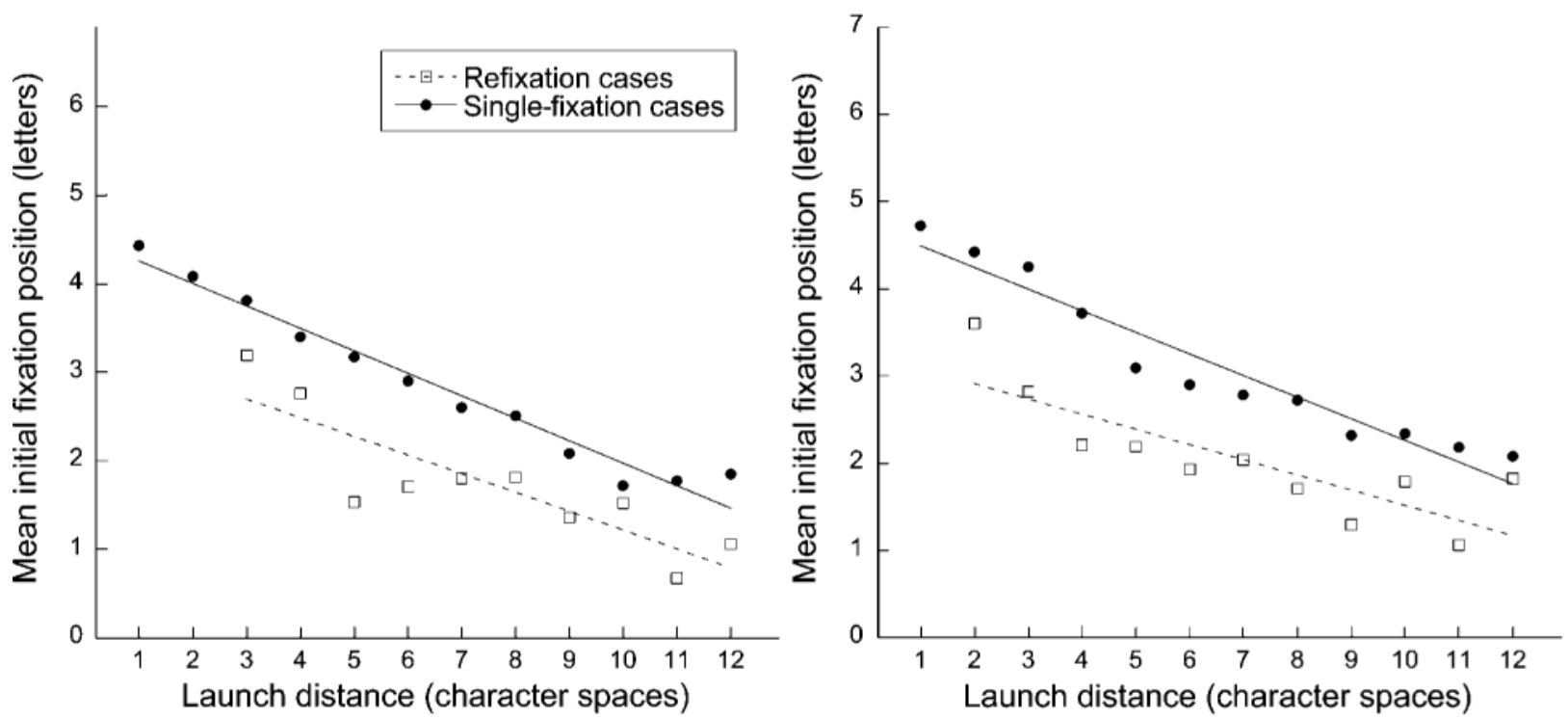

Figure 3. Mean landing position as a function of launch distance for 6-letter (left panel) and 7-letter (right panel) words, comparing single-fixation with refixation cases, with associated least-squares linear fits. Each data point represents the mean value of a minimum of 10 cases. 
spective of launch distance, indicating that there is neither a systematic range error nor an undershooting propensity for interword regressions. This finding is inconsistent with data presented in Rayner (1979). Although mean landing position was not formally analyzed, visual examination of the relevant histograms suggests that more interword regressions land to the right than to the left of center. However, comparable analyses of our own corpus of data confirm the results of Radach and McConkie; mean landing position for regressive saccades made from word $n$ to $n-1$ were located very close to word-center: $2.44,2.95$, $3.61,4.02,4.58,5.0$, and 5.69, for 4- through 10-letter word $n-1$, respectively.

Further evidence against the inevitability of undershooting is provided by Ducrot and Pynte (2002), who report that saccades directed to linguistic targets presented either to the right or to the left of fixation landed slightly left of target-center. If saccadic undershooting is an integral feature of word reading, saccades directed to leftward targets should have landed, on average, to the right of center.

\section{What Factors Could Influence the Planning of Refixations?}

We have made the case for the conflation of refixation and single-fixation saccades contributing to the PVL effect and will now explore a potential motivation for refixation planning. Although very good descriptive reports of refixation behavior during text reading have been published (McConkie et al., 1989), explanations of the circumstances that give rise to a refixation remain incomplete. One popular account states that refixations are triggered when the initial fixation lands in a nonoptimal position in the word (e.g., O'Regan, 1990); thus the aim of the refixation saccade is to bring unidentified letters into foveal vision. Other accounts are motivated by word identification considerations, which propose that the purpose of refixations is to make more letter information available. Because the perceptibility and hence the identification probability of a word falls with eccentricity (Brysbaert, Vitu, \& Schroyens, 1996), if the target word is far from the current fixation position, the likelihood of correctly identifying it is lower than if the target word is close. Refixation probabilities are indeed higher for far compared with near launch distances (Vitu et al., 1990), which is consistent with this proposal. The linguistic properties of the target word also play a role because refixation likelihood is higher for low-frequency compared with high-frequency words (McConkie et al., 1989; Rayner et al., 1996; Vitu, 1991b). Predictability is also a factor; Balota, Pollatsek, and Rayner (1985) and Vitu (1991b) report lower refixation rates for words that were highly predictable from the previous context. Oculomotor error may be responsible for some proportion of refixations: An intended interword saccade may simply fall short and so land on the currently fixated word (McConkie et al., 1988). Finally, properties of the oculomotor system have been put forward as a causal factor. The fact that refixation patterns on meaningless letter strings are broadly similar to those for real words (Vitu, O'Regan, Inhoff, \& Topolski, 1995) indicates that refixations can occur in the absence of ongoing word identification demands. Using a letter search task and stimuli consisting of strings of letters, Nazir (1991) found that even when the target letter was always the fixated letter, the probability of refixation depended on the fixation location in the string.

Through analysis of our eye movement corpus, we tested the hypothesis that a simple operationalization of the perceptibility of the target word was a major determinant of refixation planning. The relationship between refixation probability (RefixP) and two important visual predictors, word length (WL) and launch distance (LD, divided into five bins) is depicted in Figure 4A. The probability of making a refixation increases as both WL and LD increase, confirming previous research. Long words are more likely to be read with more than one fixation than are short words (e.g., McConkie et al., 1989), probably because an appropriately spaced multiple-fixation sequence permits more of the word to fall in high-acuity vision than would a single fixation. Vitu et al. (1990) found that launch distance influenced refixation probability. A long launch distance may make a refixation more likely because fewer letters are perceptible when the previous fixation is far from rather than near to the target word.

Of note from Figure 4A is that the effects of these two variables appear to be multiplicative, not additive; differences in RefixP due to WL are larger for the farther launch distances. Figure 4B displays the relationship between RefixP and the product of WL and LD, which we term the estimated perceptibility. The data are well fitted by a linear model: Pearson $r$ is 0.962 . Note that because our definition of estimated perceptibility is a composite measure, determined by both the length of the target word and its distance from fixation, it is also preferred to two separate measures on grounds of parsimony.

With this measure, we can outline a simple model of (re-)fixation planning analagous to the stochastic model of word skipping proposed by Brysbaert and Vitu (1998). In their model, the probability of skipping an upcoming word is well predicted by the estimated chances of identifying it from the current fixation position. Skipping probability depended only on the length and eccentricity of the target word and a single free parameter. Similarly, the "decision" to plan either a single fixation or a refixation sequence may be primarily influenced by low-level visual properties. On the basis of previous experience, the processor may arrive at an "educated guess" about the chances of identifying the upcoming word with a single fixation, given information about its length and any partial identification of its component letters. The estimated perceptibility of the target word thus forms the basis of a stochastic (re-)fixation planning mechanism; when this perceptibility estimate is high, there is a smaller chance of planning a multiple-fixation sequence. A nondeterministic, probabilistic model such as the one outlined here can account for why some short words receive more 

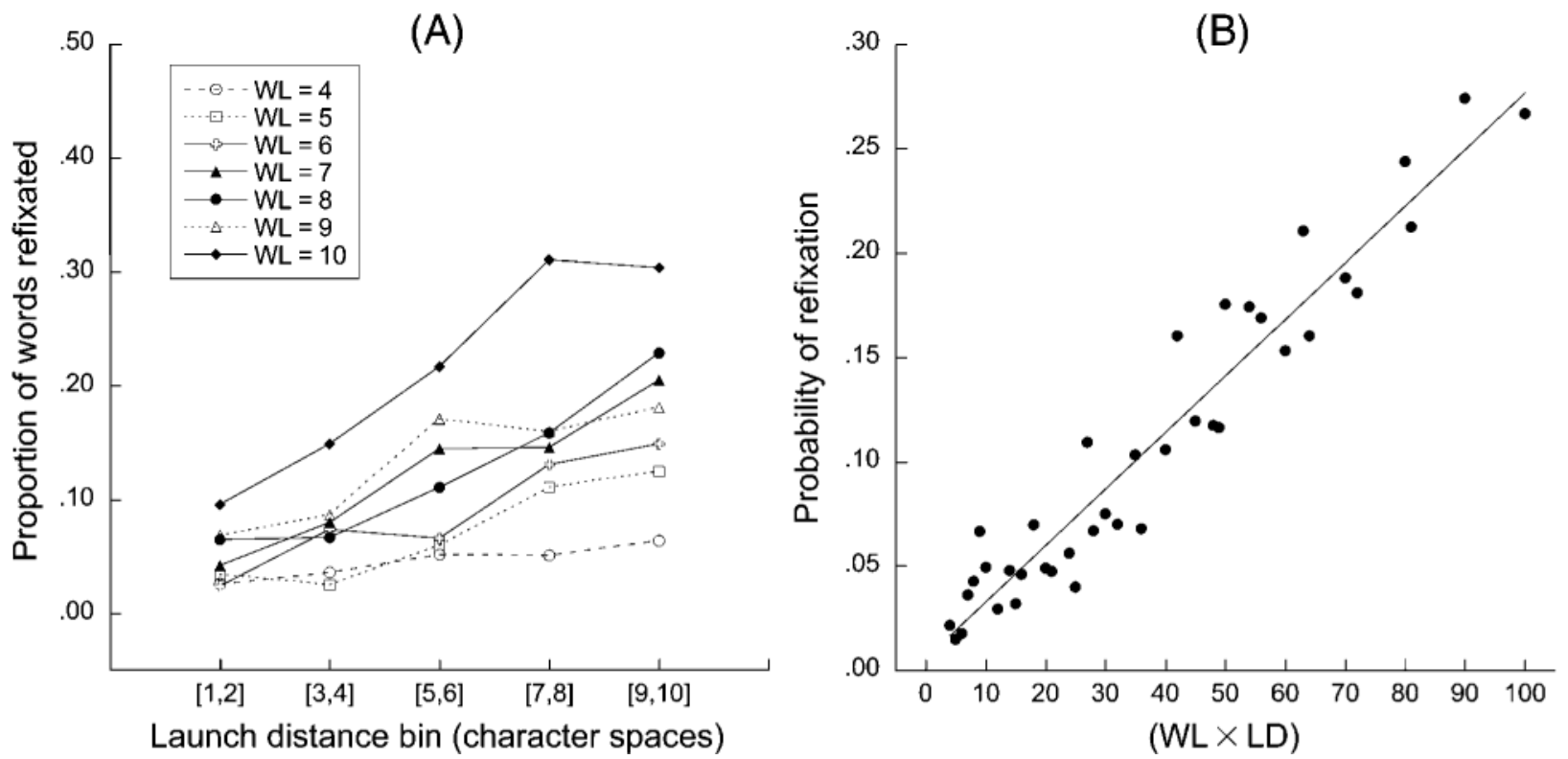

Figure 4. Refixation probability as a function of word length and binned launch distance, for 4- to 10-letter words and launch distances from $[1,2]$ through $[9,10]$ (A). Refixation probability as a function of the product of word length and launch distance (B). The line indicates the least-squares linear fit to the data.

than one fixation and some long words are fixated only once.

In summary, we have presented evidence from eye movement corpus analyses demonstrating that word identification considerations offer a viable explanation for why at least some refixations are preplanned. If the estimated perceptibility of the target word is low (primarily due to visual acuity limitations), a refixation may be planned in order to maximize the success of identifying the target word, by spreading the processing load across a sequence of fixations. If the estimated perceptibility is high, the odds are that only a single fixation is necessary. We predict that a single fixation is more likely to be planned than a refixation sequence when the perceptibility of the target word is estimated to be high than when it is estimated to be low.

As noted by Beauvillain et al. (2000), obtaining direct evidence for the preplanning of refixations under naturalistic reading conditions is extremely difficult. We instead employed a combination of corpus analysis and simulation techniques to address the consequences for the estimated word identification success of single- versus multiple-fixation reading strategies.

\section{Do Refixations Reduce Uncertainty? A Study of Observed Reading Behavior}

We have hypothesized that maximizing the chances of successful word identification provides a functional motivation for planning a refixation sequence, and that the perceptibility of the target word can be estimated as the arithmetic product of launch distance and word length. Although we have shown that refixations are more likely when estimated perceptibility is low than when it is high, we have not yet shown that refixations serve the needs of ongoing processing during reading: identifying the individual words forming the text. This was the goal of the analyses presented below.

We operationalized the likelihood of successful word identification with the information-theoretic concept of uncertainty. The visual information about a word-its component letters and length - both contribute to determining the word's identity. For instance, knowing the length and the first 4 and final letters of the word desperate unambiguously resolves its identity, since no other English 9-letter word matches this sequence. However, if only the first 3 letters are available, uncertainty is present because there are three other possible matches in the lexicon (dessicate, designate, desecrate).

This operationalization of word identification probability as uncertainty has precedence in the work of Brysbaert et al. (1996), who demonstrated that the probability of successfully recognizing a briefly presented backwardmasked 5-letter word was influenced by the informativeness of its beginning and ending trigrams. When the point of fixation was imposed on the first letter, words uniquely specified by their first 3 letters had a significantly greater likelihood of being identified than did words with a redundant beginning and unique ending. When fixation was imposed on the fifth letter, the opposite was true: Identification of words with unique beginnings was more difficult than words with unique endings.

Our account extends previous word identificationmotivated accounts of refixation behavior by bringing a key anatomical constraint into the picture: the precise vertical splitting of the fovea and consequent contralateral 
projection of the two halves of the visual field to the primary visual cortex (see Leffe, 2004, for a review of the physiological evidence for foveal splitting). The fact that the fovea is split has implications for word identification during isolated word recognition tasks (Shillcock et al., 2000), as well as for fixations made during normal reading, as letter information in the right visual field will initially be projected to the left hemisphere (LH), with letters in the left hemifield going to the right hemisphere (RH). In other research, we have found both the RH and LH uncertainty about a word's identity (i.e., computed from the letter information in the left and right hemifields, respectively) to be predictors of saccade planning behavior (McDonald \& Shillcock, in press). In left-to-right reading, letter information from a parafoveal target word is necessarily obtained from the right hemifield; our analyses therefore focus on the estimation of LH uncertainty.

We computed LH uncertainty as the entropy of the distribution of words that fitted any partial visual information; specifically, it was computed as a function of the number of words in the CELEX lexicon (Baayen, Piepenbrock, \& Gulikers, 1995) that matched the target in length and available orthographic information (the letters falling in the right hemifield). When there are two possibilities matching the partial information available, uncertainty is $\log _{2}(2)=1$ bit. When a word is completely identified, or there is only one possible lexical entry matching the partial information available, uncertainty is zero. ${ }^{2}$ As a rough estimate of the extent to which letter information can be obtained to the right and left of fixation, we assumed a conservative forward "window" of 6 characters (cf. Rayner, 1998; Underwood \& McConkie, 1985) and a backward window of 4 characters (cf. Rayner, Well, \& Pollatsek, 1980). We also assumed that partial orthographic information available from the previous fixation is trans-saccadically integrated with the letter information obtained from the current fixation (through parafoveal preview; e.g., Rayner, McConkie, \& Zola, 1980).

If the consequence of executing a refixation sequence is the reduction of uncertainty about a word's identity, on average we should find the uncertainty upon the eyes leaving a word to be lower for refixated cases compared with single-fixation cases. This prediction leads to a second, related prediction concerning the planning of a single fixation. Why are a large proportion of long words read with a single fixation when a refixation sequence would obviously permit more letters to fall into highacuity vision? It may be that, for these cases, a single fixation is "good enough" to recognize the word. So, if the refixated words in our corpus had not been refixated, but had instead received a single fixation, word identification would have been more difficult than for the singlefixation cases. "True" single fixations can thus be compared to "simulated" single fixations (i.e., multiplefixation cases in the data reclassified as single-fixation cases). We predicted that the average uncertainty upon the eyes leaving true single-fixation cases would be lower than the value for simulated single-fixation cases.
To test the first prediction, we computed the LH uncertainty of each word in our dataset at the last position fixated before the eyes left the word. As already noted, we assumed a 6-character forward window from which letters could be identified and a 15-character forward window from which low-spatial frequency visual information, such as word length, could be obtained (McConkie \& Rayner, 1975). Thus, the beginning letters of a long word could often be identified from the previous fixation and trans-saccadically combined with letters identified from the current fixation. Figure 5A plots the average LH uncertainty for words of 4-10 letters. From Figure 5A, it is clear that the mean uncertainty upon the eyes leaving a word is always lower for the refixated cases; the differences for all word lengths were statistically reliable $(t \mathrm{~s}>4.62, p \mathrm{~s}<.001)$. This was not a surprising result because the leftward-landing position of the first fixation of a refixation sequence means that the letters in the first half of the word are more likely to be identified than if a single fixation was made (which has a mean landing position close to word-center). ${ }^{3}$ Due to the informational properties of the English lexicon, words are more likely to be identified from their beginnings than from their endings (Farid \& Grainger, 1996). Right hemisphere uncertainty was also significantly lower for the refixated cases $(t \mathrm{~s}>8.65, p \mathrm{~s}<.001)$; a single fixation typically allows only the beginning of a word to be projected to the $\mathrm{RH}$, whereas a sequence of two or more successive fixations would permit more of the word to be projected to the RH.

We tested the second prediction with a simulation procedure; we replaced sequences of two or more fixations occurring on all 4- to 10-letter words in the EMBRA corpus of data with a single fixation. The location of this simulated fixation was randomly sampled from the empirical landing position distribution for nonrefixated words of the same length, from the same corpus. The two hemispheric uncertainty measures were computed as described above. It is important to note that any parafoveally available letter information from the previous fixation was not changed in the simulation procedure. The data resulting from the simulation is a good approximation of the data that would have been produced had refixated words instead received a single fixation.

Figure 5A illustrates the difference in average LH uncertainty upon the eyes' leaving the target word, comparing the true single-fixation cases with the simulated single-fixation cases. Mean uncertainty was always lower for the true singlefixation cases; this difference was statistically significant $(t \mathrm{~s}>2.54, p \mathrm{~s}<.02)$. Refixation sequences replaced by single fixations are clearly worse for reducing LH uncertainty (and hence, the estimated chances of successful word identification) than are true single-fixation cases. The situation is markedly different when RH uncertainty is computed (Figure 5B). Although refixations are clearly advantageous for minimizing the RH's uncertainty about the word's identity upon the eyes' leaving the word, there was no reliable difference between the true and simulated single-fixation cases in 

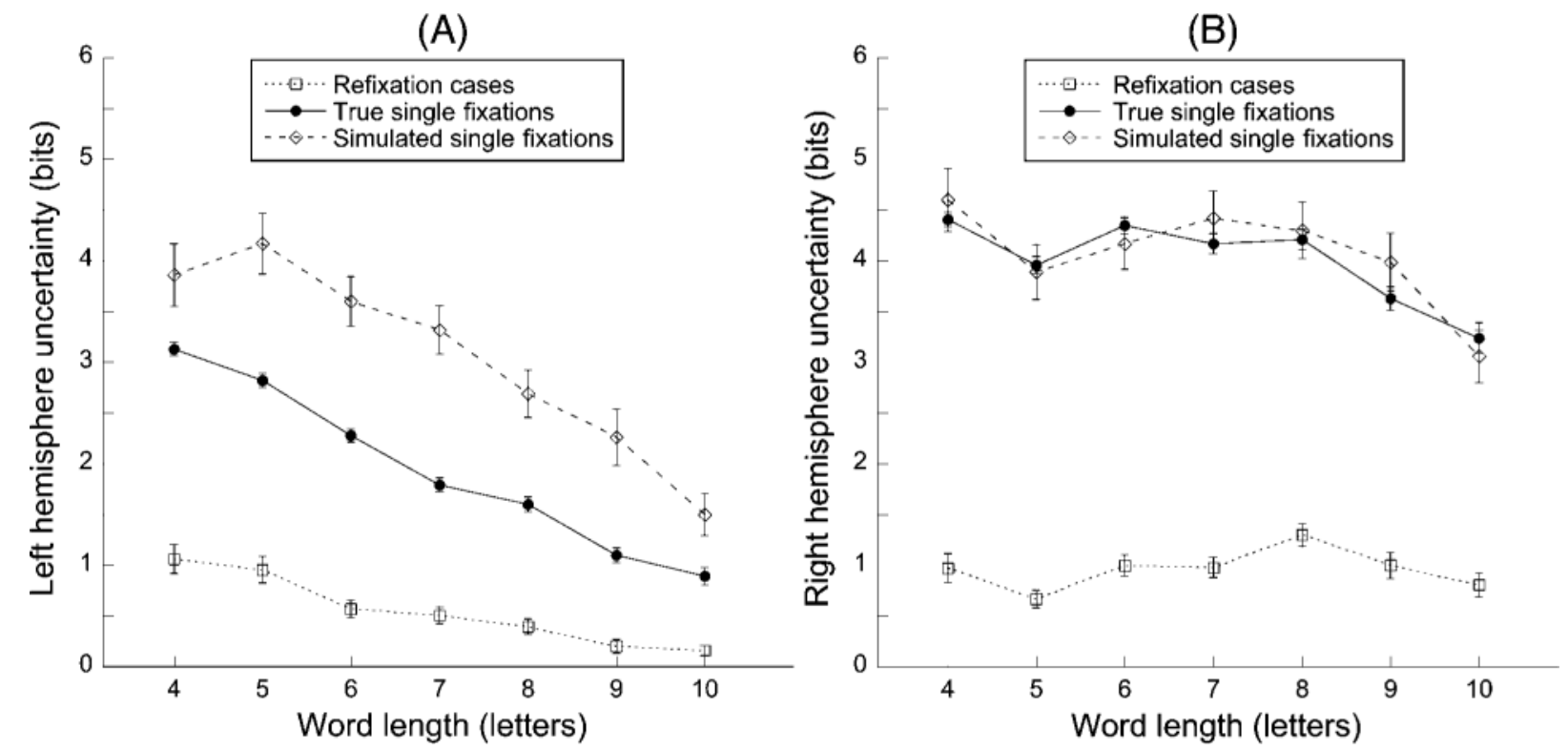

Figure 5. Mean left (A) and right (B) hemisphere uncertainty (in bits) about word identity upon leaving the target word. Refixated cases, single-fixation cases, and refixated cases replaced with a single fixation (where fixation position was randomly sampled from the empirical initial fixation position distribution for that word length) are compared. Bars indicate standard errors.

terms of estimated word identification success $(t \mathrm{~s}<1.17$, $p \mathrm{~s}>.24)$. This between-hemispheres difference in simulation results can be attributed entirely to the left-to-right reading direction of English and the associated partial identification of upcoming words through parafoveal preview. The fact that the true single-fixation cases are lower in LH uncertainty than the simulated single-fixation cases is likely because the quality of parafoveally obtained visual information made reading the target word with a single fixation viable. Because the right hemisphere can never receive advance letter information about an upcoming word when the eyes move forward through the text, parafoveal preview cannot influence the RH's confidence about the identity of the target word. Thus there is no advantage in terms of the $\mathrm{RH}$ uncertainty measure in reading the target word with a single fixation.

On the basis of the results of this simulation, we can conclude that the observed saccade planning behaviorreading even long words with a single fixation - is justified when word identification uncertainty is considered.

\section{GENERAL DISCUSSION}

We have presented the case for dividing saccades into two populations: those that are followed by a refixation and those that are followed by a saccade out of the word. When single-fixation cases are considered separately, the preferred viewing location phenomenon is attenuated, with the mean landing position falling slightly left of center for the 4- to10-letter words in the EMBRA corpus. Our results show that the generally accepted explanation for the PVL as saccadic undershooting is not nec- essarily the best account. Rather, the hypothesis that some proportion of saccades are aimed near the word beginning (between the second and third letter positions) - initiating a preplanned sequence of two or more fixations - is supported by Beauvillain and colleagues' double-step experiments, and provides an alternative explanation for the PVL. Note that the preplanning of saccade sequences represents a form of precompiled behavior that may be less computationally expensive than programming saccades online.

Beauvillain and colleagues' theory of preplanned refixations can be contrasted with theories that attribute the primary cause of refixations to ongoing processing demands (the "direct-control" model). In the latter type of model (e.g., O'Regan, 1990), the decision to refixate is assumed to take place after the initial fixation is made, and the metrics of the refixation saccade depends on factors such as the initial fixation location and word length. The primary factor triggering a refixation saccade is the location of the initial fixation: If the eyes land in an optimal or near-optimal position, a refixation is not required and an interword saccade is programmed next. Note that both models assign primary importance to lowlevel visual information, while permitting a subsidiary role for cognitive influences such as predictability and word frequency.

One way to contrast these two models with respect to their potential contributions to the PVL is that for the "preplanned" model, the leftward-shifted landing position distribution apparent for refixation cases is the consequence of (planning) a refixation saccade; for the 
direct-control model, the initial landing position is the cause of a refixation saccade. Much of the data is compatible with either hypothesis. Although the primary aim of the present article is to propose an alternative account of the PVL, our analyses point out the need to consider all of the factors potentially influencing refixation behavior during reading. We believe that the most accurate explanation of refixation behavior is likely some combination of the preplanned and direct-control models: When considering an entire set of refixation data, particular cases may be attributable to one of the two accounts. The task of future research is to elucidate the circumstances favoring one model or the other.

A recent study by Huestegge, Radach, Vorstius, and Heller (2003) was designed to examine the metrics of refixation saccades with a more realistic sentence reading paradigm. During the primary saccade to the target word, the entire line of text was displaced, and landing position distributions were compared with the distributions obtained in a control condition where no displacement occurred. Huestegge et al.'s results were inconsistent with the constant refixation saccade size reported by Beauvillain and colleagues; instead, results were compatible with either refixation saccades being triggered and computed as a function of the (actual) landing position in the word, or with the planning and subsequent revision of the refixation saccade size (cf. Vergilino-Perez \& Beauvillain, 2004). More research is required to estimate the relative proportions of planned and triggered refixations that occur during natural reading.

In the corpus analyses presented above, we have certainly overestimated the number of preplanned refixation sequences by treating all refixated cases as a homogenous population. However, because at present it is very difficult to distinguish preplanned refixations from unplanned refixations (e.g., those triggered by ongoing processing demands), and consequently there is no straightforward way of estimating the proportion of refixations that are preplanned (but cf. Doré-Mazars, Vergilino-Perez, \& Collins, 2003), we have necessarily aggregated all refixated cases into the same group. This simplification means that the effect on the PVL represents an upper bound on the expected influence of preplanned refixations.

\section{Canceling a Refixation Saccade}

The results of Beauvillain et al.'s (2000) double-step experiments showed that refixation frequency was also dependent on landing position. An opposite-direction stimulus displacement results in the initial landing position distribution moving rightward, closer to the wordcenter (or OVP), with the consequence that refixation frequency decreased (and the frequency of saccades directed toward the next word increased). This indicates that although the amplitude of the refixation saccade is preplanned, the refixation saccade itself can be canceled and an interword saccade executed instead. We suggest that refixation sequences are preplanned in many cases, with the likelihood of cancellation of the refixation sac- cade being contingent on the actual position in the word that is initially fixated and the ability of the word to be identified on the basis of visual information available from this landing position. Canceling a saccade may be less costly in processing terms than programming an additional fixation on the target item; if so, it seems plausible that the eye guidance system programs sequences of saccades when targeting long words in particular.

\section{Computational Models of Refixations}

One of the first attempts to implement refixation behavior in a computational model of reading was made by Reilly and O'Regan (1998). They modeled the triggering of a refixation saccade as a random process based upon the two-parameter quadratic equation of McConkie et al. (1989). Refixation probability in Reilly and O'Regan's model was determined by fixation position and word length only.

Early versions of the E-Z Reader model (Reichle, Pollatsek, Fisher, \& Rayner, 1998) explained refixations as planned events subject to cancellation, but assumed that the refixation saccade was planned at the onset of the initial fixation on the word and whether or not the refixation was executed was determined by a race between the lexical processing of the fixated word and the programming of the refixation saccade. However, the race assumption was problematic in that first fixation durations predicted by the model were far too short. The most recent version, E-Z Reader 7 (Reichle, Rayner, \& Pollatsek, 2003), also considers refixation behavior as a stochastic process; in the model a refixation program (which can be canceled) is initiated with a probability that depends only on the length of the fixated word.

The SWIFT model of reading (Engbert, Longtin, \& Kliegl, 2002) takes a different approach to modeling refixations, in that intraword saccades are treated no differently than interword saccades. Words are selected as saccade targets according to a random function whose parameters are determined by the degree of lexical activation of each of the candidate words in the model's "perceptual span." The currently fixated word is included as one of these candidates.

Although all three models are able to fit the empirical refixation data reasonably well, they are deficient in that they do not account for important factors that also influence refixation likelihood, such as the eccentricity of the target word (Vitu et al., 1990). A comprehensive model of eye movement control in reading should also consider Beauvillain et al.'s (2000) evidence for the preplanning of refixation saccades in reading-like tasks, and the role that the preplanning of refixation sequences would play in a computational implementation.

\section{Conclusions}

This article has put forward an alternative explanation for the preferred landing location phenomenon in reading. The left-of-center landing position distributions describing the PVL can be explained as the mixing of two 
populations of saccades, aimed at distinct target locations. We have suggested that refixation sequences are preplanned in many cases, and proposed that the planning of (re-)fixation saccades is consistent with a stochastic model that exploits low-level visual information to estimate the chances of successfully identifying the target word with a single fixation. Eye movement data collected during the reading of natural text are consistent with this hypothesis by showing that uncertainty about word identity upon the eyes leaving the word is in fact lower for refixated words than for words receiving a single fixation. In this respect, the behavior of the eye guidance system appears to be optimal, given constraints due to visual acuity, the composition of the lexicon (the existence of orthographic competitors), and a uniform reading direction.

\section{REFERENCES}

BaAyen, R. H., Piepenbrock, R., \& Gulikers, L. (1995). The CELEX lexical database [CD-ROM]. Philadelphia: University of Pennsylvania, Linguistic Data Consortium.

Balota, D. A., Pollatsek, A., \& Rayner, K. (1985). The interaction of contextual constraints and parafoveal visual information in reading. Cognitive Psychology, 17, 364-390.

Beauvillain, C., Vergilino, D., \& Dukic, T. (2000). Planning twosaccade sequences in reading. In A. Kennedy, R. Radach, D. Heller, \& J. Pynte (Eds.), Reading as a perceptual process (pp. 327-354). Amsterdam: Elsevier.

BECKER, W. (1989). Metrics. In R. H. Wurtz \& M. E. Goldberg (Eds.), The neurobiology of saccadic eye movements (pp. 13-61). Amsterdam: Elsevier.

BRysbaert, M., \& VitU, F. (1998). Word skipping: Implications for theories of eye movement control in reading. In G. Underwood (Ed.), Eye guidance in reading and scene perception (pp. 125-147). Amsterdam: Elsevier.

Brysbaert, M., Vitu, F., \& Schroyens, W. (1996). The right visual field advantage and the optimal viewing position effect: On the relation between foveal and parafoveal word recognition. Neuropsychology, 10, 385-395.

Clark, J. L., \& O'Regan, J. K. (1999). Word ambiguity and the optimal viewing position in reading. Vision Research, 39, 843-857.

Deutsch, A., \& RAYNER, K. (1999). Initial fixation location effects in reading Hebrew words. Language \& Cognitive Processes, 14, 393421.

Doré-Mazars, K., Vergilino-Perez, D., \& Collins, T. (2003). Are there two populations of refixations in the reading of long words? Commentary on Reichle et al., "The E-Z Reader model of eye movement control in reading: Comparisons to other models." Behavioral \& Brain Sciences, 26, 480-481.

Ducrot, S., \& Pynte, J. (2002). What determines the eyes' landing position in words? Perception \& Psychophysics, 64, 1130-1144.

Dunn-Rankin, P. (1978). The visual characteristics of words. Scientific American, 238, 122-130.

ENGBERT, R., LONGTIN, A., \& Kliegl, R. (2002). A dynamical model of saccade generation in reading based on spatially distributed lexical processing. Vision Research, 42, 621-636.

FARID, M., \& GRAINGER, J. (1996). How initial fixation position influences visual word recognition: A comparison of French and Arabic. Brain \& Language, 53, 351-368.

FINDLAY, J. M. (1982). Global processing for saccadic eye movements. Vision Research, 22, 1033-1045.

Huestegge, L., RADACH, R., Vorstius, C., \& Heller, D. (2003, August). On the metrics of refixation saccades in normal reading. Paper presented at the 12th European Conference on Eye Movements. Dundee, Scotland.

HYÖNÄ, J. (1995). Do irregular letter combinations attract readers' at- tention? Evidence from fixation locations in words. Journal of Experimental Psychology: Human Perception \& Performance, 21, 6881.

KAPOUla, Z. (1985). Evidence for a range effect in the saccadic system. Vision Research, 25, 1155-1157.

KAPOULA, Z., \& RoBINSON, D. A. (1986). Saccadic undershoot is not inevitable: Saccades can be accurate. Vision Research, 26, 735-743.

LEFFE, A. (2004). A historical review of the representation of the visual field primary cortex with special reference to the neural mechanisms underlying macular sparing. Brain \& Language, 88, 268-278.

Legge, G. E., Klitz, T. S., \& TJan, B. S. (1997). Mr. Chips: An idealobserver model of reading. Psychological Review, 104, 524-553.

McConkie, G. W., Kerr, P. W., ReddiX, M. D., \& ZoLA, D. (1988). Eye movement control during reading: I. The location of initial eye fixations on words. Vision Research, 28, 1107-1118.

McConkie, G. W., KerR, P. W., RedDix, M. D., Zola, D., \& JaCobs, A. M. (1989). Eye movement control during reading: II. Frequency of refixating a word. Perception \& Psychophysics, 46, 245-253.

McConkie, G. W., \& RAYNER, K. (1975). The span of the effective stimulus during a fixation in reading. Perception \& Psychophysics, 17, 578-586.

McDonald, S. A., \& SHILlCock, R. C. (2003). Low-level predictive inference in reading: The influence of transitional probabilities on eye movements. Vision Research, 43, 1735-1751.

MCDonald, S. A., \& SHILlCoCK, R. C. (in press). Anatomical constraints on eye fixation behavior: The implications of foveal splitting for saccade planning in reading. Vision Research.

NAZIR, T. A. (1991). On the role of refixations in letter strings: The influence of oculomotor factors. Perception \& Psychophysics, 49, 373389.

O'Regan, J. K. (1981). The convenient viewing position hypothesis. In D. F. Monty, R. A. Senders, \& J. W. Senders (Eds.), Eye movements: Cognition and visual perception (pp. 289-298). Hillsdale, NJ: Erlbaum.

O'Regan, J. K. (1990). Eye movements and reading. In E. Kowler (Ed.), Eye movements and their role in visual and cognitive processes (pp. 395-453). Amsterdam: Elsevier.

O’Regan, J. K., Lévy-Schoen, A., Pynte, J., \& Brugaillère, B. (1984). Convenient fixation location within isolated words of different length and structure. Journal of Experimental Psychology: Human Perception \& Performance, 10, 250-257.

RADACH, R., \& MCCONKIE, G. (1998). Determinants of fixation positions in reading. In G. Underwood (Ed.), Eye guidance in reading and scene perception (pp. 77-100). Oxford: Elsevier.

RAYNER, K. (1979). Eye guidance in reading: Fixation location within words. Perception, 8, 21-30.

RAYNER, K. (1998). Eye movements in reading and information processing: 20 years of research. Psychological Bulletin, 124, 372-422.

RAYNer, K., MCConkie, G. W., \& Zola, D. (1980) Integrating information across eye movements. Cognitive Psychology, 12, 206-226.

RAYNER, K., \& MORRIS, R. K. (1992). Eye movement control in reading: Evidence against semantic preprocessing. Journal of Experimental Psychology: Human Perception \& Performance, 18, 163-172.

RAYNER, K., SERENO, S. C., \& RANEY, G. E. (1996). Eye movement control in reading: A comparison of two types of models. Journal of Experimental Psychology: Human Perception \& Performance, 22, 11881200.

Rayner, K., Well, A. D., \& Pollatsek, A. (1980). Asymmetry of the effective visual field in reading. Perception \& Psychophysics, 27, 537-544.

Reichle, E. D., Pollatsek, A., Fisher, D. L., \& Rayner, K. (1998). Toward a model of eye movement control in reading. Psychological Review, 105, 125-157.

Reichle, E. D., Rayner, K., \& Pollatsek, A. (2003). The E-Z Reader model of eye-movement control in reading: Comparisons to other models. Behavioral \& Brain Sciences, 26, 445-526.

ReILly, R. G., \& O'Regan, J. K. (1998). Eye movement control during reading: A simulation of some word-targeting strategies. Vision Research, 38, 303-317.

ShILlCOCK, R., Ellison, T. M., \& Monaghan, P. (2000). Eye-fixation behavior, lexical storage, and visual word recognition in a split processing model. Psychological Review, 107, 824-851. 
Underwood, G., Clews, S., \& Everatt, J. (1990). How do readers know where to look next? Local information distributions influence eye fixations. Quarterly Journal of Experimental Psychology, 42A 39-65.

UNDERwood, N. R., \& McConkie, G. W. (1985). Perceptual span for letter distinctions during reading. Reading Research Quarterly, 20, 153-162.

Vergilino, D., \& Beauvillain, C. (2000). The planning of refixation saccades in reading. Vision Research, 40, 3527-3538.

Vergilino-Perez, D., \& Beauvillain, $\overline{\mathrm{C}}$. (2004). The ability of the saccadic system to change motor plans in scanning letter strings. Psychonomic Bulletin \& Review, 11, 332-337.

VITU, F. (1991a). The existence of a centre of gravity effect in reading. Vision Research, 31, 1289-1313.

VITU, F. (1991b). The influence of parafoveal preprocessing and linguistic context on the optimal landing position effect. Perception \& Psychophysics, 50, 58-75.

Vitu, F., McConkie, G., Kerr, P., \& O'Regan, J. K. (2001). Fixation location effects on fixation durations during reading: An inverted optimal viewing position effect. Vision Research, 41, 3513-3533.

Vitu, F., O'Regan, J. K., InHOFF, A. W., \& TopolsKi, R. (1995). Mindless reading: Eye-movement characteristics are similar in scanning letter strings and reading texts. Perception \& Psychophysics, 57, 352364.
Vitu, F., O’Regan, J. K., \& Mittau, M. (1990). Optimal landing position in reading isolated words and continuous text. Perception \& Psychophysics, 47, 583-600.

\section{NOTES}

1. Beauvillain et al. (2000) report that refixation likelihood also depended on the initial fixation position; when the target displacement was in the same direction as the saccade (meaning that the actual landing position moved closer to word-beginning), refixation likelihood increased; when it was in the opposite direction (moving the landing position closer to word-center), refixation likelihood decreased.

2. Related measures have been investigated by Clark and O'Regan (1999) and Legge, Klitz, and Tjan (1997).

3. Of subsidiary interest from Figure $5 \mathrm{~A}$ is the observation that average LH uncertainty decreases as word length increases. This effect of WL on LH uncertainty is statistically reliable for both single-fixation and refixation cases $[F(1,6)=37.43, p<.001 ; F(1,6)=17.54$, $p<0.01$, respectively]. On average, uncertainty decreases with length because there are in general fewer orthographic matches for partially identified longer words than for partially identified short words.

(Manuscript received March 14, 2003; revision accepted for publication November 10, 2003.) 